Paper:

\title{
Spinal Information Processing and its Application to Motor Learning Support
}

\author{
Mihoko Otake ${ }^{*, * *}$, and Yoshihiko Nakamura ${ }^{* * *}$ \\ *Division of Project Coordination, University of Tokyo \\ 5-1-5 Kashiwa-no-ha, Kashiwa, Chiba 277-8568, Japan \\ E-mail: otake@cb.k.u-tokyo.ac.jp \\ **PRESTO program, Japan Science and Technology Agency \\ *** Graduate School of Information Science and Technology, University of Tokyo \\ 7-3-1 Hongo, Bunkyo-ku, Tokyo 113-8656, Japan \\ [Received January 28, 2005; accepted June 14, 2005]
}

\begin{abstract}
We designed motor learning support for acquiring motor skills involving neural mechanisms. We should be able to acquire neural information by analyzing whole-body muscle data, because the nervous system controls the musculoskeletal system and lengths and forces information is fed back to the nervous system. Motor information is calculated by mapping motioncapture data on to a musculoskeletal human model. Neural information represents the set of motor information on the muscles innervated by the arbitrary spinal cord segment. Neural information processing is proposed which calculates correlation among the neural information. We demonstrate the effectiveness of our proposal by experimental results of "kesagiri".
\end{abstract}

Keywords: motor learning, muscle innervation, simulation, spinal cord, human behavior analysis

\section{Introduction}

Expert players and masters use repetitive exercises to build advanced motion skills. How can this be done most efficiently? Motion capture is used to measure physical movement and acquire three-dimensional locations of specific physical sites. Joint angle and muscle lengths are calculated by mapping this data onto a musculoskeletal human model [1-4]. The fact that the solution is not necessarily uniquely determined remains a problem, but once boundary conditions are determined, tension in wholebody muscles can be determined $[5,6]$. Systems such as SIMM [7] (Delp et al.) and AnyBody [8] (Rasmussen et al.) have been marketed to analyze and simulate musculoskeletal human model movement. Even if beginners generate the same muscle activity pattern as experts, they cannot produce the same movement because of differences in individual physical structure. Muscle activity patterns must suit the physical structure of the individual. Also, assume the muscle activity patterns generating a target movement are acquired artificially by optimized calculation. For robots, if the acquired solution is given

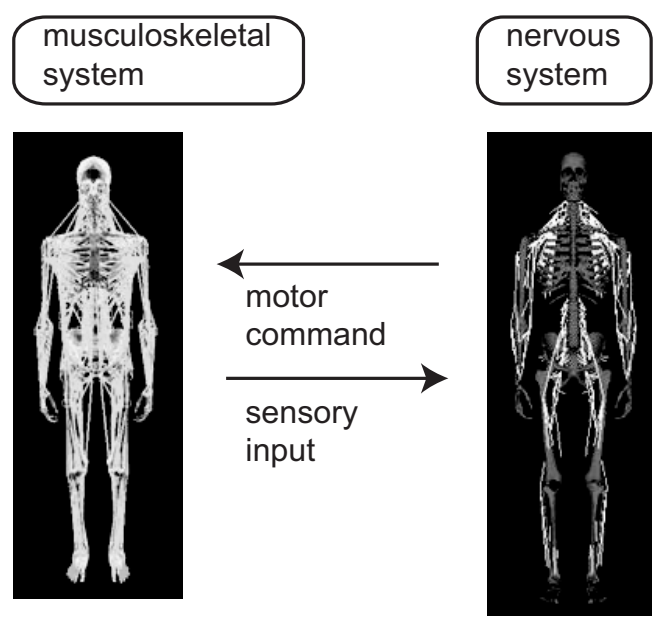

Fig. 1. Information flow between the musculoskeletal system and the nervous system of the human body.

as the target and used for control, the target movement is generated. In contrast, human beings have sufficient muscle force to generate a muscle activity pattern, the issue becomes one of whether the targeted nerve activity pattern can be produced - something that, unfortunately, cannot be done consciously. As shown by the anatomical structure of the spinal cord, nerve circuits are locally integrated and massed segments are bundled from the top for driving. If the combination of low-dimensional characteristics of nerve activity patterns and objective evaluation of movement are made clear, nerve activity patterns could be generated consciously to implement targeted movement.

Here we present an overview of conventional movement analysis. Most studies used parts of whole-body movement that consolidate coordinated movement, such as the hand or foot location, or the joint angle of the elbow or knee, to compare movements based on movement measurement. Previous studies also compared the activity of each muscle, such as myopotential and muscle length. Sakurai et al. compared a tennis veteran and a beginner based on the displacement in peak time of muscle activity [9]. Simply comparing the results of coordinated move- 


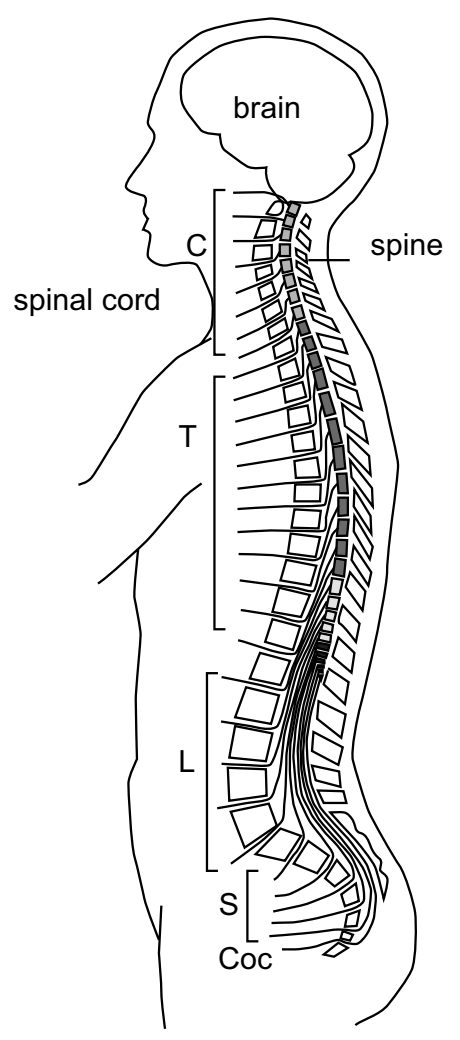

Fig. 2. Central nervous system of humans consisting of brain and spinal cord. The spinal cord is divided into 31 segments that correspond to attachments of groups of nerve roots. The skeletal muscles are classified by the innervated nerves.

ment, i.e., postures of the hand or foot, however, prevented partial displacement of the entire body from being detected. Muscle tension found by electromyography was restricted by the number of electromyograph channels. Describing whole-body movement using only part of the muscles distributed throughout the body, failed to clarify the degree of local coordination. Muscle length acquired by mapping motion-capture data onto a musculoskeletal model had hundreds of dimensions. As a result, major muscles were selected for comparison and information about a variety of muscles was not available enough to extract global and local movement characteristics.

Our purpose is to provide information processing that extracts characteristics needed for monitoring and supporting motor learning to improve motor skill. Specifically, motor information is converted to neural information based on the anatomical structure of the nervous system in the spinal cord (Fig.1). Their characteristics are calculated and their correspondence to the evaluation are accumulated. We determined local values such as dimension or correlation of neural information, together with global values such as the phase differences between the set of neural information through calculation. We measured repetitive exercises to clarify the characteristics acquired. We discuss how to utilize acquired characteristics to support motor learning.

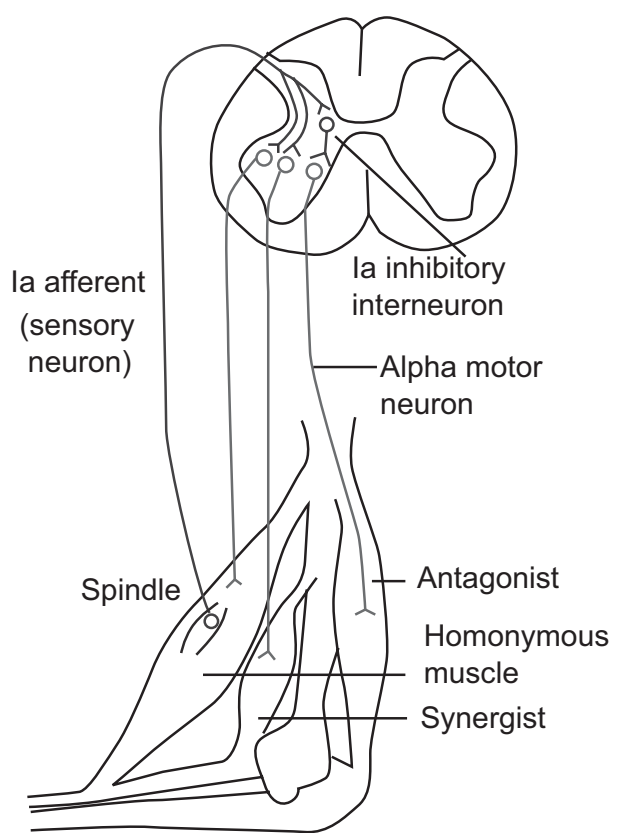

Fig. 3. Simple reflex pathway comprising sensory neurons from muscle spindles to the spinal cord, motor neurons from the spinal cord to the muscles, and interneurons between the sensory motor neurons [10].

\section{Calculation of Spinal Neural Information Based on Motor Measurement}

\subsection{Anatomical Structure of Spinal Cord $[11,12]$}

The nervous system consists anatomically of central and peripheral nervous systems. The central nervous system generally brings to mind the brain, but consists of the brain and spinal cord. The peripheral nervous system consists of cranial nerves connecting brain directly to organs and spinal nerves connecting the spinal cord to organs. The spinal cord and spinal nerves are generically called the spinal nervous system. Organs are controlled by different nerves, and are classified by the innervated nerves. The 31 pairs of human spinal nerves consist of 8 pairs of cervical nerves (C), 12 pairs of thoracic nerves (T), 5 pairs of lumbar nerves (L), 5 pairs of sacral nerves (S), and a single pair of coccygeal nerves (Coc). These nerves pass through and depart from the clearance between spinal bones (Fig.2).

The muscle that operates a joint in cooperation with an agonist is called the synergist, while that operates the joint in opposition is called the antagonist. In order to generate ordered motion, groups of muscles must contract as their synergists contract and antagonists relax. Nerves are thus connected in the spine so that if an agonist is excited, its antagonist is suppressed to excite the synergist (Fig.3). The resulting movement is coupled and the degree of freedom (DOF) is smaller than the number of muscles. 

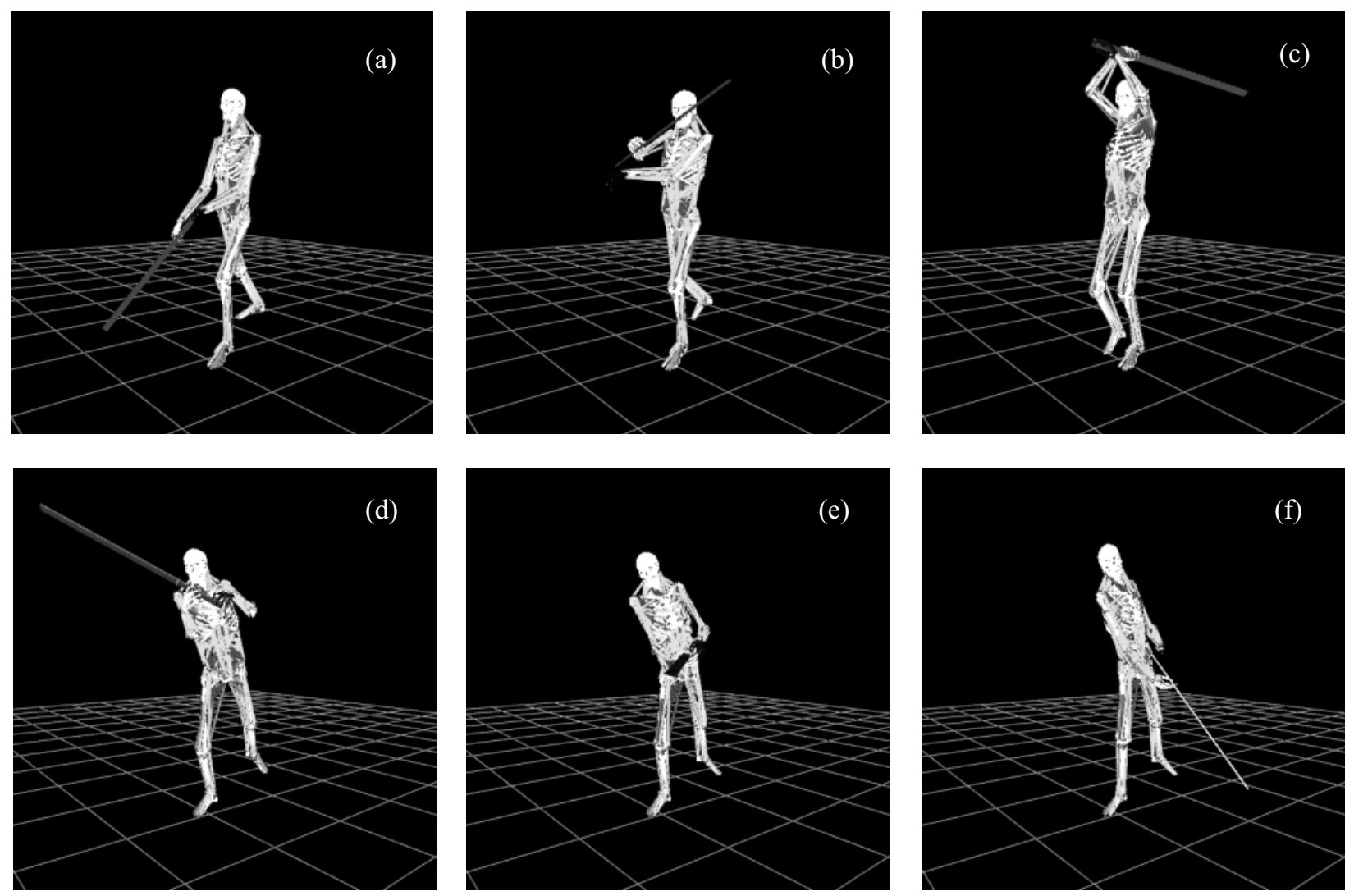

Fig. 4. Snapshot of sword swinging "kesagiri" motion: Motion capture data are mapped onto the musculoskeletal model.

\subsection{Sensory Motor Organs and Control Nerves}

A muscle spindle senses muscle elongation and is parallel to extrafusal muscle fiber. The Golgi tendon organ senses force generated by a muscle. Other sensory motor organs include the joint receptor responding to torque applied to a joint and the nociceptor responding to joint pain.

\subsection{Calculation of Spinal Neural Information}

We focused on the spinal nervous system controlling most skeletal muscles and on the spinal nerve connecting spinal cord and sensory motor organs. They convert motor information to neural information bundled by spinal cord segments based on the muscle control structure. Fig.5 shows the spatiotemporal pattern of neural information via (a) the musculocutaneous nerve and (b) the obturatorius nerve during "kesagiri" (left or right up-to-down diagonal cutting movement) (Fig.4). We extracted muscle length data on a muscle controlled by these nerves and normalized it with the muscle length when standing. The value is represented by intensity. Numbers (1) and (2) in Fig.5 refer to the trial numbers.

\section{Measurement and Calculation of Whole- Body Movement}

\subsection{Measurement of Motion and Calculation of Muscle Movement}

We used optical motion capture to measure the threedimensional location of markers in kesagiri. We used inverse kinematics calculation to find the joint angle and muscle length of the musculoskeletal human model and inverse dynamics calculation to find muscle tension. Time-series data was acquired each $33 \mathrm{~ms}$. The muscle length was divided by the length when standing and normalized. The musculoskeletal human model [4] consists of 366 muscles, 91 tendons, 34 ligaments, 56 cartilages, and 53 bone groups.

\subsection{Kesagiri}

Kesagiri is the left or right up-to-down diagonal cutting movement by a sword named for the traditional Buddhist priest's left-over-right wrapped "stole" or "kesa" and indicating a diagonal cut from the wrap crossing just below the neck and across the breast down to the waist as follows:

1) The line of sight is maintained toward the direction the sworder is facing, with the haft of the sword positioned along the frontal median line. The left foot 


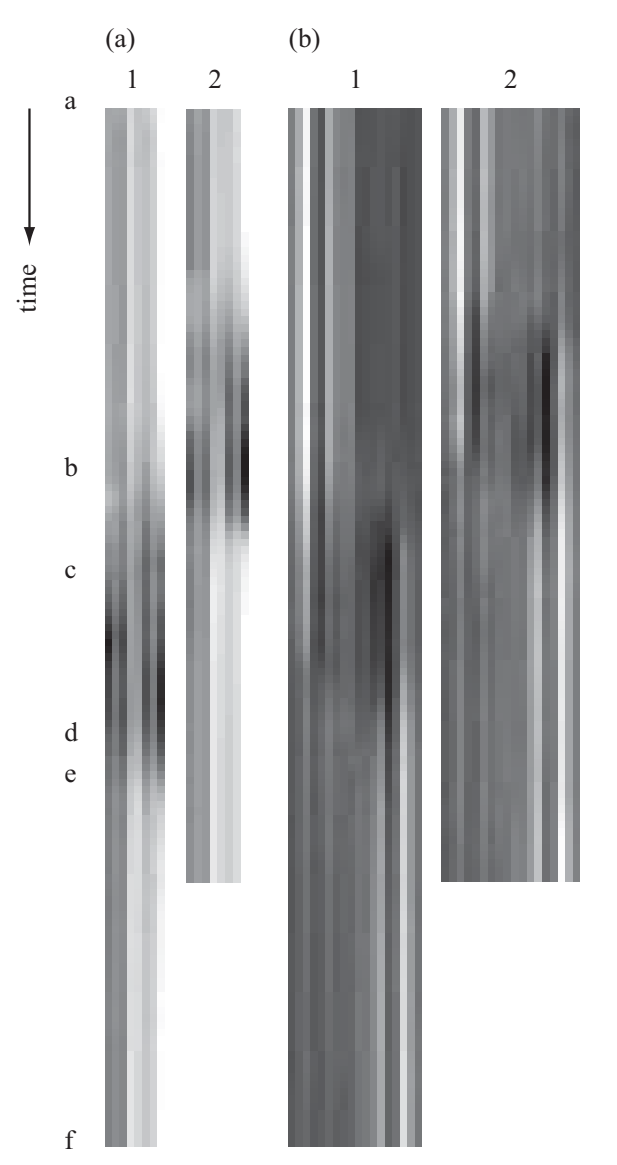

Fig. 5. Spatiotemporal pattern of the neural information at (a) musculo cutaneus nerve and (b) obturatorius nerve during "kesagiri" motion. The numbers 1 and 2 indicates the trial numbers. The characters a to $\mathrm{f}$ correspond to the snapshot of Fig.4. The innervating muscles are: (a) corfig:correlillustacobrachialis, biceps brachii, and brachialis muscle; (b) obturatorius, adductor longus, adductor brevis, pectineus, grachilis, adductor minimus, adductor magnus from center to both sides.

is set forward, the right foot back (vice versa for a left-handed sworder), so the sword cuts diagonally forward (Fig.4(a)).

2) The sword is swung up to the left above the head. The cutting edge moved forward diagonally right. The feet change position as the opponent is cut down (Fig.4(b) to (d)).

3) The sword is swung down from upper right straight to lower left to maximize its velocity at the front (Fig.4(d) to (f)). The shoulders should be as relaxed as possible to accelerate the sword tip under the force of gravity.

4) As the sword reaches the lower left, it is abruptly halted with the right foot positioned forward and the left foot back (Fig.4(f)).

As a typical coordinating movement involving the entire body, kesagiri requires much practice. It is evaluated

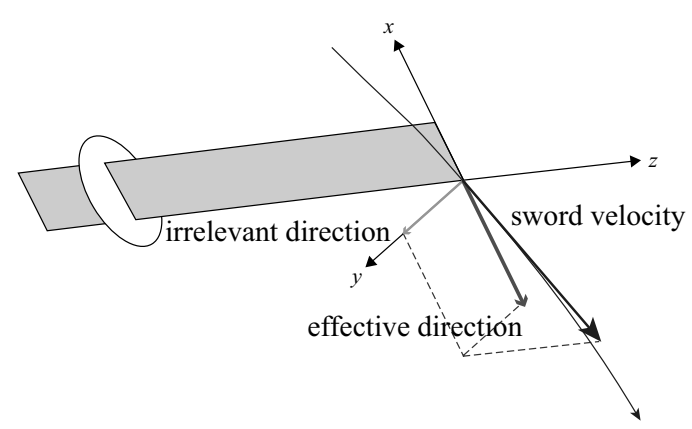

Fig. 6. Decomposition of sword velocity into effective and irrelevant directions.
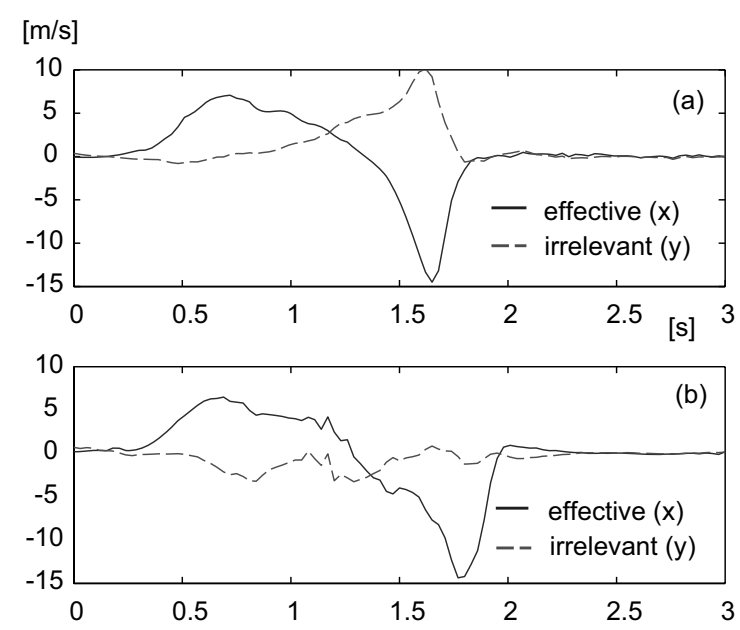

Fig. 7. Effective and irrelevant velocities of the sword tip; objective evaluations were (a): 1, (b): 5 .

based on (a) the location of the starting position; (b) the location of the end point; (c) the direct trajectory advance connecting the starting and end points; (d) proper wholebody relaxation, particularly the arm; and (e) the dynamic stability of the entire body. Unless otherwise noted, the data handled is sensory information acquired by measuring kesagiri.

\subsection{Calculation of Evaluation Values}

We focused on the direct trajectory advance in (c) above, considered most important for cutting down an opponent. Specifically, velocity is broken down into the effective component parallel to the sword blade and the irrelevant (unstable) component perpendicular to the blade to compare their maximum values (Fig.6). If the $\mathrm{x}$-axis is taken from the blade to the back, the $y$-axis perpendicular to the blade and toward the right from the back, and the $\mathrm{z}$-axis from the haft of the sword to the tip, the effective component is $x$ and the ineffective component $y$. If the effective component is greater and the irrelevant component smaller, the sword tip is evaluated as having its direction along the trajectory and the score of direct advance higher. Fig.7 plots sword tip velocities for low and high evalua- 

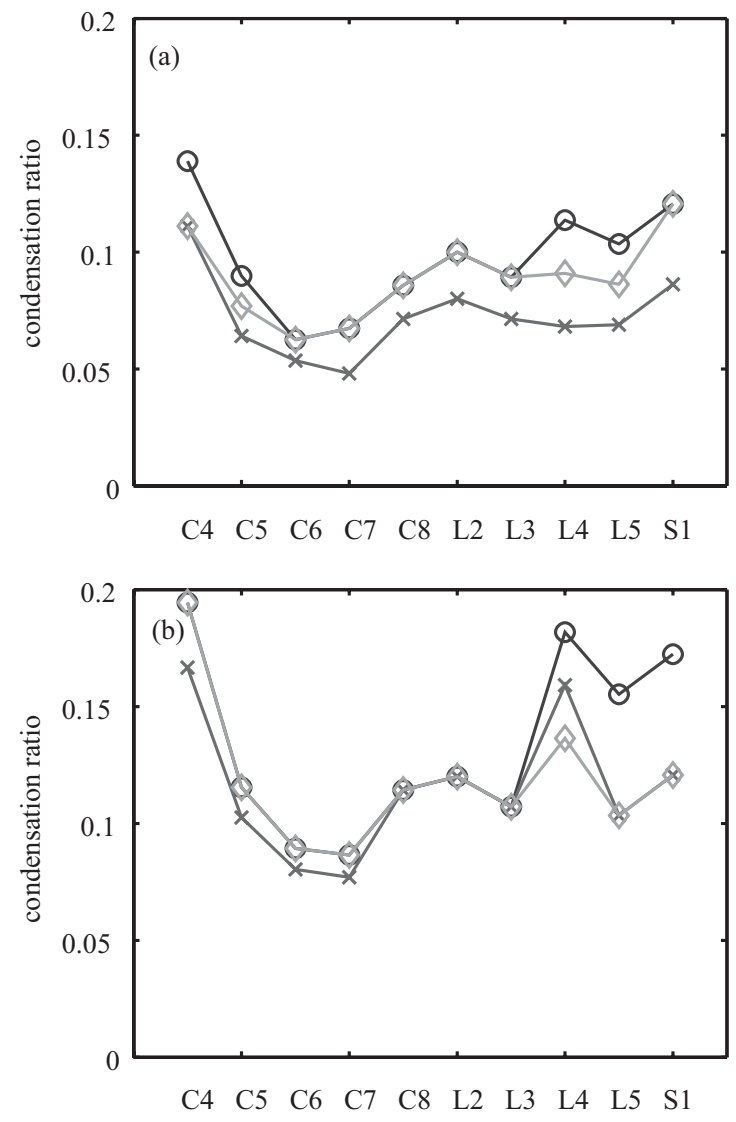

Fig. 8. Condensation ratio at each section of the spinal cord during (a) "kesagiri" and (b) kick motion.

tions of direct trajectory advance. Maximum values of the y component are 10.1 and $0.844 \mathrm{~m} / \mathrm{s}$, while sword tip velocities are almost the same and the $\mathrm{x}$ component peaks at -14.5 and $-14.4 \mathrm{~m} / \mathrm{s}$.

\section{Spinal Neural Information Processing}

\subsection{Dimension of Spinal Neural Information}

In order to examine motor coordination in the same segment of the spinal cord, the dimension of somatic neural information is found by analyzing principal components. Specifically, the number of elements is calculated for accumulated contribution to exceed 0.8 . To determine how the dimension is reduced compared to the original DOF, a reduction ratio is calculated dividing the dimension by the number of elements. The smaller this reduction ratio, the higher the degree of coordination.

We selected kesagiri and a kick in the middle as examples of whole-body coordination and conducted three experiments for each. We found the dimension and reduction ratio for neural information near cervical ( $\mathrm{C} 4$ to $\mathrm{C} 8$ ) and lumber (L2 to L5, S1) enlargement of the spinal cord (Fig.8). These enlargements control the upper and lower limbs. The dimension of neural information was 4 to 7 in (a)

(b)
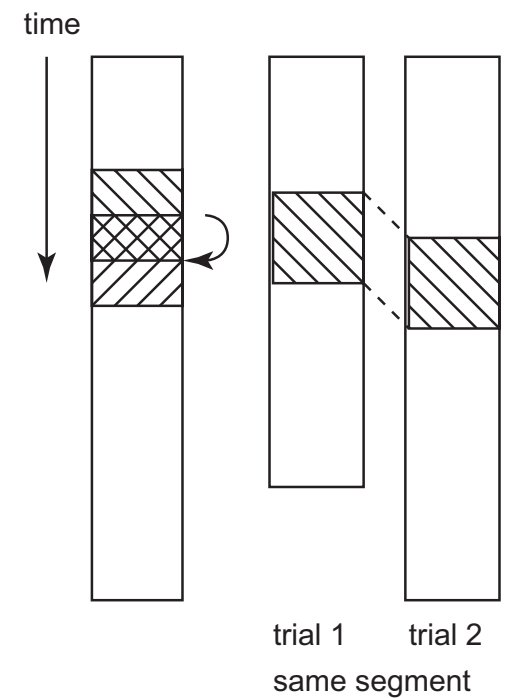

Fig. 9. Calculation of temporal change and phase contrast in spinal neural information based on correlation.

each spinal cord segment for kesagiri. A reduction ratio that divides the dimension by the number of elements was, about 0.06 , particularly near $\mathrm{C} 6$ and $\mathrm{C} 7$, about 0.1 at other sites. Similarly, for the kick in the middle, the dimension of neural information was 6 to 10, while the reduction ratio was 0.2 at $\mathrm{C} 4$ and 0.1 at other sites. Compared to the kick, kesagiri has a smaller reduction ratio. A specifically large difference is found at $\mathrm{C} 4$ and L4, each of which has a small reduction ratio and a small difference at $\mathrm{C} 6$ and $\mathrm{C} 7$. For the kick, the upper limb (C4 to $\mathrm{C} 8$ ) is constant, and a large variation is seen on the lower limb (L2 to S1). Coordination changes at these locations where the reduction ratio changes, i.e., spinal cord segment greatly changes the timing of driving muscle. Our results represent the amount of information required to approximate the time series of data with the linear vector. We found that somatic neural information during whole-body motion was extremely reduced for each spinal cord segment and that the degree of coordination was high. For two types of experimental movement, we found that the condensation ratio was at the same level, about 0.1 .

\subsection{Temporal Change in Spinal Neural Informa- tion}

The pattern of time $t$ to $t+N$ is extracted from the spatiotemporal pattern of neural information, and the correlation with time $t+1$ to $t+N+1$ is calculated (Fig.9(a)). A temporal change in correlation is acquired with one step later time pattern. The value subtracting one from correlation is assumed to be variation. Correlation is used here because if the difference is determined conventionally, it is adversely affected by noise.

We found afferent neural information (muscle length information) sent from the muscle spindle of the entire 

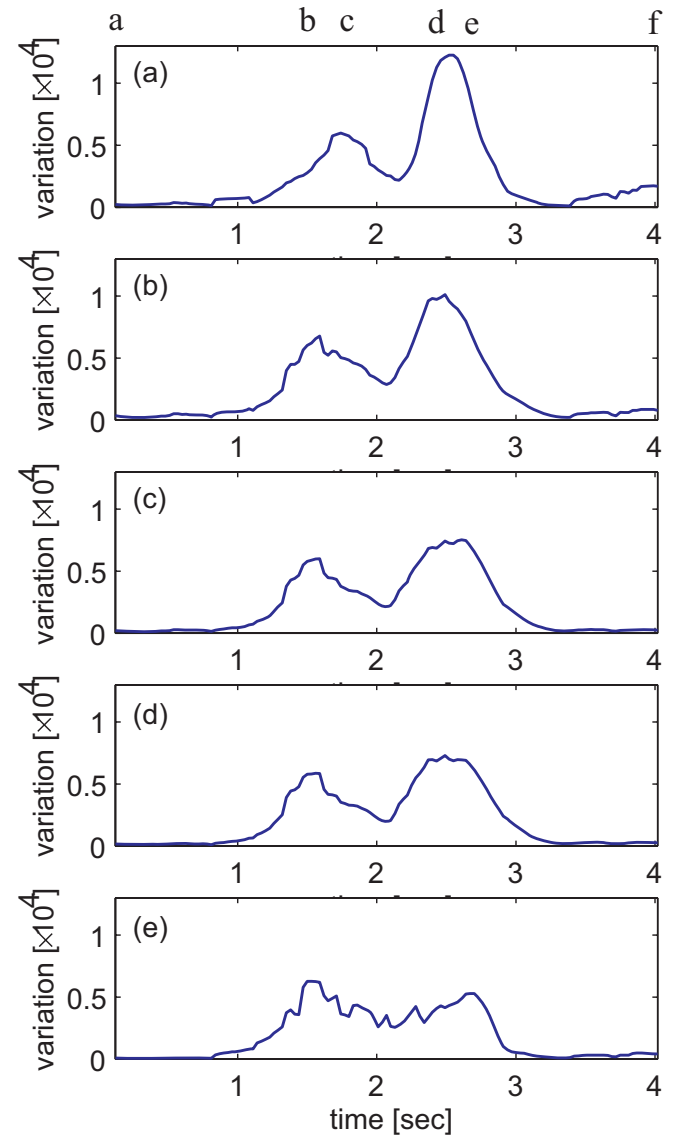

Fig. 10. Temporal change of spinal neural information at cervical enlargement: (a)C4, (b)C5, (c)C6, (d)C7, (e)C8. The characters a to $f$ correspond to the snapshot of Fig.4.

body to the spinal cord by calculation. The time window size was set to $N=9$. Fig.10 and Fig.11 show temporal changes in neural information near cervical enlargement (C4 to C8) and lumbar enlargement of the spinal cord (L2 to L5, S1). These enlargements control the upper and lower limbs. A comparison of changes in cervical and lumbar enlargement show that those of cervical enlargement exceeds those of lumbar, and both have two peaks. Upper positioning at cervical enlargement causes the last half to have a greater peak than the first half. In contrast, the lower positiononing at lumber enlargement causes the last half has a greater peak. Changes are smoother at lumber enlargement than at cervical enlargement. The first half peaks sooner at cervical enlargement except at $\mathrm{C} 4$, while the last half peaks earlier at lumbar enlargement. We found that muscular activity synchronized and changed for each spinal cord segment and that synchronization timing differs slightly.

\subsection{Correlation and Phase Difference in Spinal Neural Information}

This section details procedures for calculating the correlation and phase difference of the spatiotemporal pattern of neural information (Fig.9(b)). Let the spatiotemporal
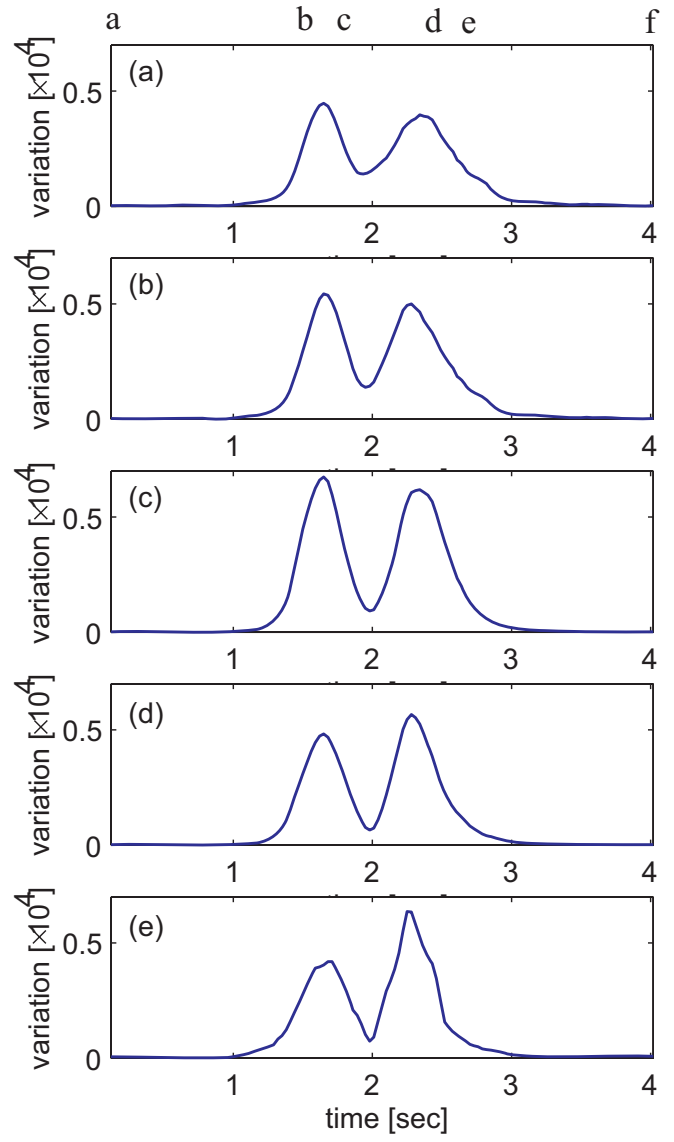

Fig. 11. Temporal change of spinal neural information at lumbar enlargement: (a)L2, (b)L3, (c)L4, (d)L5, (e)S1. The characters a to $f$ correspond to the snapshot of Fig.4.

patterns of the first trial as source data, while those of the following trial as the target data. Computational procedure is as follows:

1) Take a template $x(i)$ from the source data of arbitrary period from time $i$ to $i+N$. The partial target data of arbitrary period from time $j$ to $j+N$ are taken as $y(j)$. The lengths of the source and target data are $i_{\text {end }}$ and $j_{\text {end }}$, respectively.

2) Correlation of the $x(i)$ and $y(j)$ are calculated.

$$
f(x(i), y(j))=\frac{x(i) \cdot y(j)}{|x(i)||y(j)|}
$$

The maximum value of $f(x(i), y(j))$ among every $y(j)\left(0<j<j_{\text {end }}-N\right)$ and the corresponding time $j_{\max }$ were stored for each template $x(i)$. Correlation is from -1 to +1 , and 1 for complete agreement, if the size agrees and the direction is opposite, -1 is used.

3) Maximum correlations $f\left(x(i), y\left(j_{\max }\right)\right)$ for each template $x(i)$ are obtained by substituting 0 through $i_{\text {end }}-N$ into $i$. 

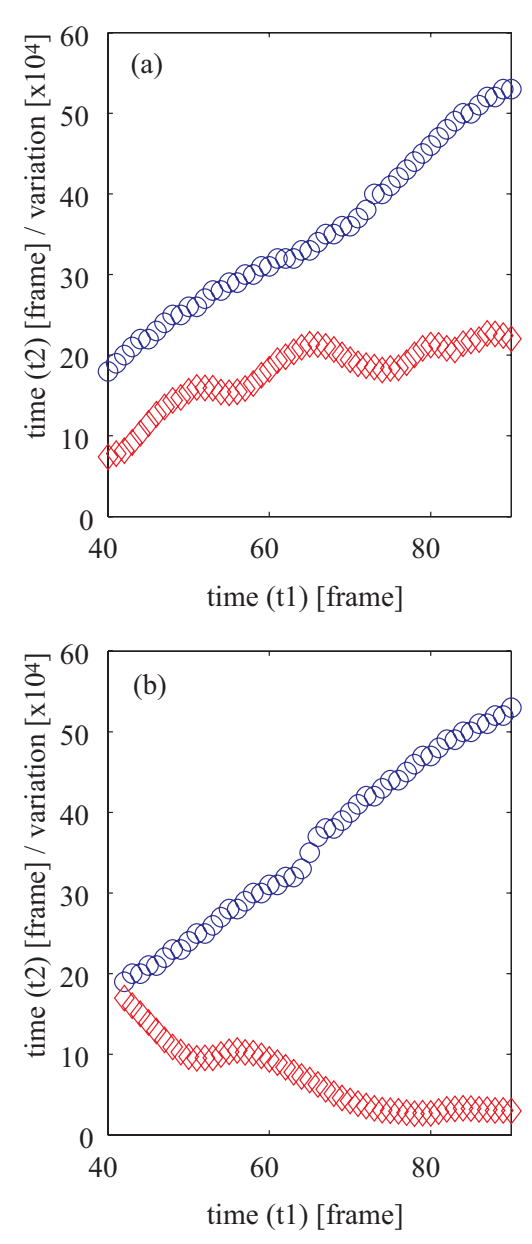

Fig. 12. Corresponding time and variation between the neural information of (a) the fifth cervical nerve (C5) and (b) the second lumbar nerve (L2) during the two trials for the "kesagiri" motion. Corresponding times are plotted by circles and variations are plotted by rhombuses.

With this method, we can compare the differences of each neural pattern and corresponding time. In this study, the size of the template $N$ was 9 . Results were plotted in the following manner:

1) Draw a horizontal axis for the time of source data, and a vertical axis for plotting the corresponding time of target data and their differences. Variation $1-f(x, y)$ was plotted since the correlation $f(x, y)$ was nearly equals to 1 . Note that maximum value of correlation is 1 and its minimum is -1 .

2) Make lines between the corresponding times of horizontal axis drawn in parallel: top axis for the time of source data and a bottom axis for the time of target data. The purpose of this time chart is to make clear the phase difference of each trial in the same segments.

3) Draw horizontal axes in parallel: top axis is for the time of target data of one segment, and bottom axis is for the one of another segment. In this way, relative phase differences between segments are obtained.

\subsubsection{Correlation and Corresponding Time of Neural Information}

Figure 12 plots correlation and the corresponding time of neural information on spinal nerves. These correspond to the fifth cervical nerve (C5) and the second lumbar nerve (L2). The horizontal axis shows the time of trial 1 , while the vertical axis shows the corresponding time of trial 2 and the correlation between these times. Correlation was so great that a variation was taken and multiplied by $10^{4}$ for plotting. This means that the greater the value, the greater the pattern difference. Correlation is plotted by rhombuses and corresponding times by circles.

The corresponding time plot (Fig.12(a)) for the fifth cervical nerve (C5) is smooth with a slope below 1 in the first half. Locations after 75 frames have a slope of almost 1. Compared to trial 1 , trial 2 indicates that the first half has quick change. In contrast, the second lumbar nerve (L2) (Fig.12(b)) has a sharp slope between 65 frames and 70 frames and a smooth slope at other times. Compared to 1 , trial 2 has a smaller velocity where the slope is sharp.

\subsubsection{Phase Difference in Neural Information}

Figure 13 plots the timing chart for two trials after the corresponding time is read from correlation. For Fig.13(a) and Fig.13(b), the upper stage of the horizontal axis is the time for trial 1 , while the lower stage is corresponding time for trial 2. Fig.13(c) shows the corresponding time between different nerves of trial 2 based on the time of trial 1 of Fig.13(a) and Fig.13(b). The upper stage of the horizontal axis in Fig.13(c) is the time of the fifth cervical nerve (C5), while the lower stage of the horizontal axis is the corresponding time of the second lumbar nerve (L2). The corresponding time was taken each 5 frames.

The timing misalignment of the fifth cervical nerve (C5) focuses on the first half (Fig.13(a)). 20 frames from 50 to 70 frames in trial 1 correspond to 10 frames from 26 to 36 frames in trial 2, indicating half of the time. In other words, a similar pattern appeared at double speed. For the latter half, 36 to 46 frames correspond to 70 to 80 frames in trial 1 at the same speed. The timing misalignment of the second cervical nerve (L2) is slight found in both the first and latter halves (Fig.13(b)). 65 to 70 frames in trial 1 correspond to 35 to 40 frames in trial 2 at the same speed on average. Before and after them, the time for 11 frames is needed for 15 frames, and 7 frames for 10 frames. From the corresponding time of C5 and L2 in trial 2 (Fig.13(c)), C5 activity begins later than $\mathrm{L} 2$ and proceeds for such a delay. In contrast, the latter half proceeds later than L2. We found that the spatiotemporal pattern for each spinal cord segment was similar and that the generated timing was different. Trial 1 had a high evaluation, but trial 2 had a low evaluation. The cause of a difference in the evaluation value was made possible to represent at neural information level. 
(a)

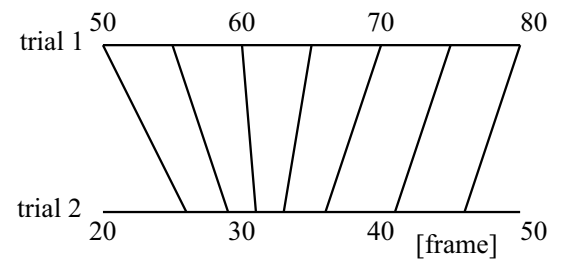

(b)

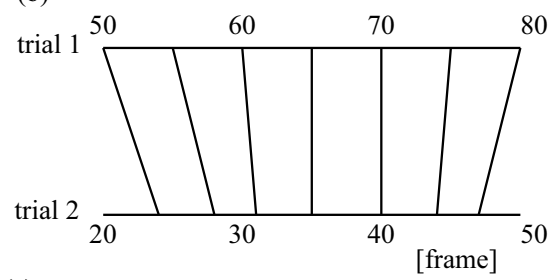

(c)

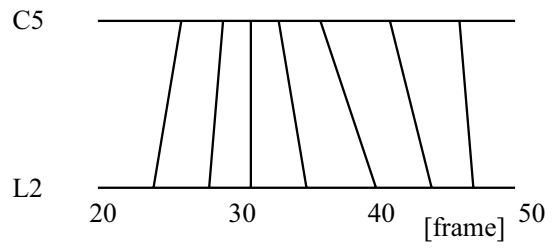

Fig. 13. Temporal diagram of the neural information of (a) the fifth cervical nerve (C5) and (b) the second lumbar nerve (L2) during the two trials for the "kesagiri" motion. Phase difference between the neural information at C5 and L2 is plotted in (c).

\subsection{Segmental and Inter-Segmental Coordination of the Spinal Neural Information}

\subsubsection{Calculation of Spinal Cord Segmental Coordi- nation}

Figure 14 shows how to calculate spinal cord segmental coordination, implemented by the correlation between reference and target data. Both data assume the spatiotemporal pattern of neural information in different spinal cord segments, extracted from the same motor data. They extract the muscle length data governed by the spinal cord segment and normalize it with the muscle length when standing for matrix arrangement. Timeseries data of muscular motor information for one muscle is extracted from reference data as the template and the correlation is calculated for that time-series data for each target data. Templates extracted from reference data should be shifted one by one, meaning that the correlation is calculated between all neural information in reference and target data. This detects the coordination of motor information between muscles controlled by a certain nerve. The higher correlation means the higher coordination between muscles and the lower correlation means the lower coordination. Acquired coordination is arranged in a matrix as shown in the figure. The number of neural data elements is not necessarily consistent nor the number of nerves the same, making it possible to calculate the correlation between different spinal segments.

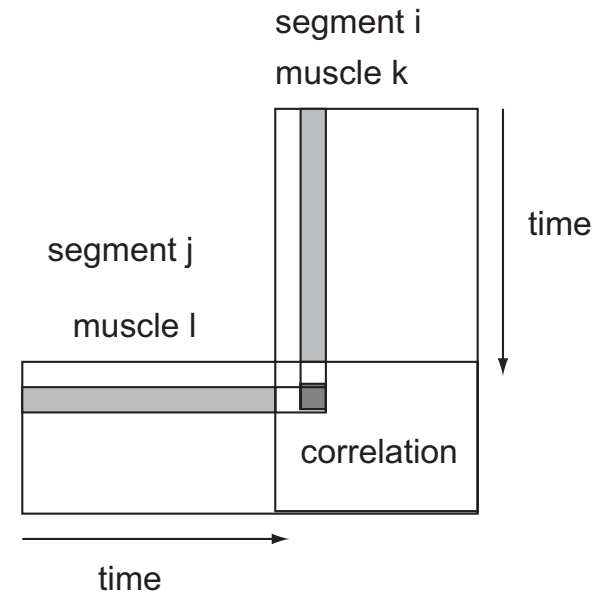

Fig. 14. Segmental coordination matrix between the segment $i$ and $j$ of neural information: each element of matrix is the correlation of muscular information innervated by the segments.

\subsubsection{Calculation of Coordination Between Spinal Cord Segments}

Next we discuss how to calculate the coordination between spinal cord segments using calculated results of correlation in the spinal cord segment. Vertical and horizontal axes of the inter-segmental coordination matrix are spinal cord segment names and the average of correlated values between corresponding segments is stored. The value of a mesh is the average of the mesh values of the intraspinal cord segment coordination matrix specified by a set of motor data and spinal cord segments. This process is repeated for all combinations of spinal cord segments. As mentioned, it is possible to calculate the condition of a local and global coordination by calculating oordination hierarchically for each spinal cord segment.

\subsubsection{Analytical Experiment of Coordinated Motion}

Figure 15 shows how the intrasegmental coordination of spinal cord is arranged at the C5 segment. C5 controls the upper body muscles such as the greater pectoral muscle, brachial muscle, and levator scapulae muscle. This indicates that the greater the intensity, the higher the coordination. The intensity of each point represents the coordination of motor information between muscles innervated by the same segment. There is a difference even in the same spinal cord segment, but coordination is high as a whole and minimum correlation was 0.949. Fig.16 shows the correlation representing the intersegmental coordination of spinal cord in a matrix. Even if the spinal cord segment location differs, there may be high coordination. When coordination between far segments is high, the command from the brain is considered synchronized. 


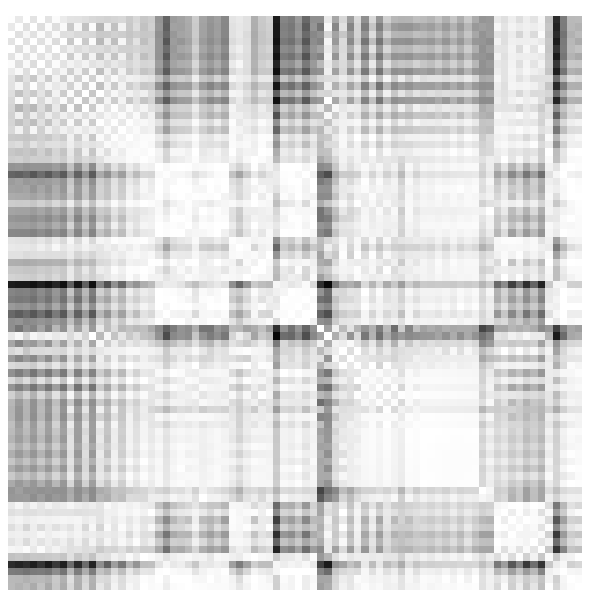

Fig. 15. Segmental coordination matrix among the segment C5 during "kesagiri” motion.

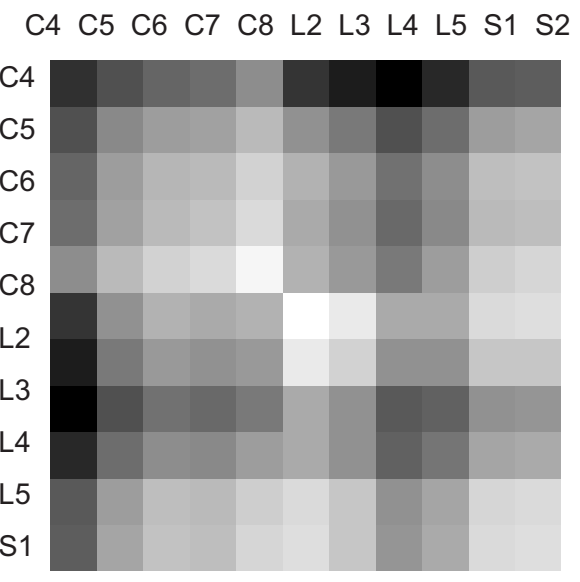

S2

Fig. 16. Intersegmental coordination matrix between the segment C4-C8, L2-L5, S1-S2 during "kesagiri” motion.

\section{Motor Learning Support}

Factors preventing implementation of the target motion are divided largely into the following three:

1) Resultant motion cannot be properly sensed.

2) Even if motion is sensed, how to correct it is unknown.

3) Even if how to correct motion is known, muscles cannot act as intended.

Correspondence between sets of objective evaluation and neural data and their comparison at the level of neural data contributes to the elimination of the factors at the first and second stages. The following procedures make characteristic data found by the proposed method helpful for motor learning support. Trials by a beginner in the motor

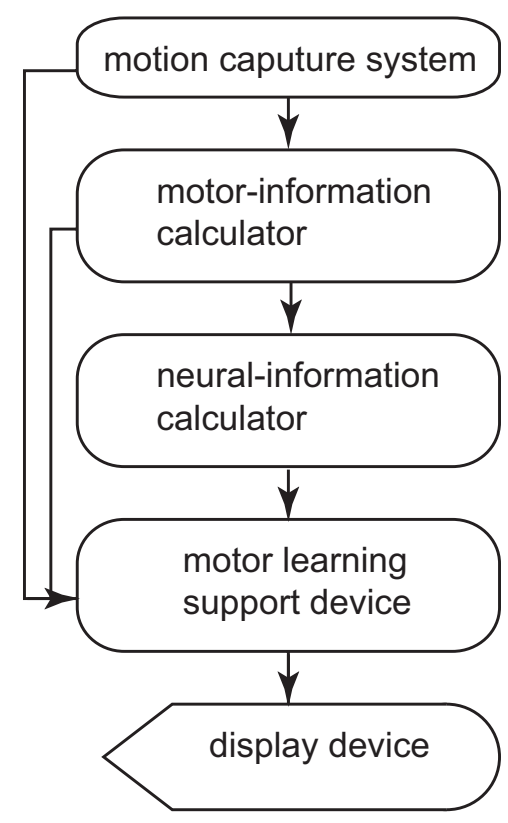

Fig. 17. Flow chart of the motor learning support system comprising motion capture system, motor-information and neural-information calculator, motor learning support device, and display device.

learning process are accumulated with progress in repetitive learning and evaluated, and then the beginner's highest trial is summarized. The objective evaluation differs for motion types, but it includes, for example, the direct trajectory advance for the kesagiri when a wooden sword is raised while stepping one step and swung down diagonally. The beginner can know when objective evaluation is high and correspondence to the coordinated condition to implement it. Repetitive trials should be made to bring them closer together.

The system configuration is shown in Fig.17. Motor learning support includes a motion-capture device, a motor information calculator, a neural information calculator, and motor learning support. The motion-capture device measures and stores the $3 \mathrm{D}$ location of the body. The motor information-calculator calculates the length and generative force (motor information) of motor organs such as muscles, tendons and ligaments from results measured by the motion-capture device and stores motor information in memory. Based on the structural function model of the human nervous system, the neural information-calculator converts motor information acquired by the motor information-calculator to neural information and stores it. The motor learning support device extracts features from motor information acquired by the motor information-calculator and neural information by the neural information-calculator and stores motor feature information and neural feature information. It also references stored motor information, neural information, motor feature information, and neural feature information and combines them with motor information acquired by the motor information-calculator or neural in- 
formation by the neural information-calculator for processing to find and store motor feature information and neural feature information. The display unit in the neural information-calculator or motor learning support device is used for presentation. The system supports the beginner in finding an achievable neural activity pattern by making the above motor feature information and neural feature information correspond to objective evaluation of movement results in the motor learning process, accumulating them, and presenting them to the beginner.

\section{Discussions}

Studies focusing on the neuromuscular system are well known in walking robot control [13-17] and estimation of arm posture from surface EMG signals $[18,19]$. We have proposed extracting motor features based on the structure of the human neuromuscular system by dividing the whole-body motion pattern into basic patterns by nerve. We do not handle the physiological parameter of the nervous system as in studies of the walking neural circuit $[20,21]$, and use only the segmental structure of the neural system to pick up information. We normalized a reduction ratio with the number of control nerves for each spinal cord segment. Other normalization with a number of degrees of joint freedom may exist. Since we focused on coordination between upper and lower limbs, we did not separate left and right for calculation, but there is a variation of this separation.

We used measured muscle length information, but sensitivity is adjusted by the $\gamma$ motor neuron. Thus, even if the length or extension velocity is the same, signals with the same strength may not always be sent from the muscle spindle to the spinal cord. Acquired spatiotemporal patterns represent neural information from motor organs, but note that they are not always consistent with strict neural signal strength, but rather represent muscle length information acquired by integrating afferent signals sent from the muscle spindle to the spinal cord and efferent signals from the spinal cord to the muscle. It is important to have successfully structured information given by signals difficult to acquire by direct measurement of the brain or nerves. We did not use information on muscle tension, because many assumed boundary conditions are required at a step in calculating the distribution of muscle tension based on joint torque acquired from inverse dynamics calculation. We used muscle length and muscular extension velocity because we can calculate these variables without any assumption. The information processing we proposed is applicable to muscle tension information in the same way.

We use the anatomical structure of the spinal cord to read body motor information as neural information for processing and present results directly to the beginner. It may be possible to convert neural information once more to information that the beginner easily intuits for more effective support of motor learning.

\section{Conclusions}

Conclusions of this paper are as follows:

- We proposed method for analyzing neural information through motion measurement. We have presented a way that extracts four features. Experimental data was processed to demonstrate its effectiveness.

- We classified muscular motion data for each innervating spinal cord segment in order to obtain the neural information based on the anatomical structure of the spinal cord.

- Firstly, we calculated the dimension of neural information and confirmed that it was extremely reduced.

- Secondly, we calculated temporal variation in neural information. The peak size and phase differences were found between neural information at different segments.

- Thirdly, correlation was calculated between local patterns of neural information. Corresponding times were found between different trials from correlation to compare phase differences generated by similar patterns.

- Fourthly, we calculated the coordination between muscles innervated by the same segment and also between different segments of the spinal cord.

- Finally, we proposed motor learning support method that makes use of the neural information features acquired above.

The proposed method is to be included into the motor learning support system comprising motion capture system, musculo-skeletal model and nervous system model. They convert motion capture data into neural information. With such a system, we can record pairs of neural information and objective data such as the speed and straightness of the sword swinging. The learner will be benefited by comparing the subjective data and internal perspective of self motion in the nervous system. Motion regulation process is seen by comparing extracted features when the same person performs a repeated practice. Analysis of tuning patterns by experts benfits the development of training to approach their techniques. Future work includes modeling of neurons, sensory and motor cells and regulation mechanisms among them. Motion measurement is the traditional method, however, this will be the powerful way for measurement and analysis of neural activities during motor learning, which is different from the direct recording of the neural signals or brain imaging method.

\section{Acknowledgements}

This work was supported by a Japan Science and Technology Agency Grant for PRESTO program "Development of the Bilateral Multiscale Neural Simulator" (PI: Mihoko Otake). 


\section{References:}

[1] N. Yamazaki, and K. Hase, "Development of three-dimensional whole body musculoskeletal model for various motion analyses," JSME International Journal, Series C, Vol.40, pp. 25-32, 1997.

[2] T. Komura, and Y. Shinagawa, "Attaching physiological effects to motion-capture data," in Proceedings of Graphics Interface, 2001, pp. 27-36.

[3] D. G. Lloyd, and T. S. Buchanan, "Strategies of muscular support of varus and valgus isometric loads at the human knee," Journal of Biomechanics, Vol.34, pp. 1257-1267, 2001.

[4] Y. Nakamura et al., "Dynamic computation of musculo-skeletal human model based on efficient algorithm for closed kinematic chains," in Proceedings of the 2nd International Symposium on Adaptive Motion of Animals and Machines, 2003.

[5] D. Davy, and M. Audu, "A dynamic optimization technique for predicting muscle forces in the swing phase of gait," Journal of Biomechanics, Vol.20, pp. 187-201, 1987.

[6] M. Kaplan, and J. Heegaard, "Predictive algorithms for neuromuscular control of human locomotion," Journal of Biomechanics, Vol.34, pp. 1077-1083, 2001.

[7] S. L. Delp, and P. J. Loan, "A computational framework for simulating and analyzing human and animal movement," IEEE Computing in Science and Engineering, Vol.2, pp. 46-55, 2000

[8] J. Rasmussen et al., "Anybody - a software system for ergonomic optimization," in Fifth World Congress on Structural and Multidisciplinary Optimization, 2003.

[9] S. Sakurai et al., "Muscle activity and performance accuracy of the smash stroke in badminton with reference to skill and practice," J. Sports Science, Vol.18, pp. 1-14, 2000

[10] E. R. Kandel, J. H. Schwartz, and T. M. Jessell, "Principles of neural science," 4th eds., McGraw-Hill, New York, 2000.

[11] J. Bossy, "Atlas of Neuroanatomy and Special Sense Organs," W. B. Saunders, 1970

[12] S. G. Waxman, "Correlative Neuroanatomy," 22nd eds., a LANGE medical book, 1995.

[13] H. Kimura, S. Akiyama, and K. Sakurama, "Realization of dynamic walking and running of the quadruped using neural oscillator," Autonomous Robots, Vol.7, pp. 247-258, 1999.

[14] S. Miyakoshi, G. Taga, Y. Kuniyoshi, and A. Nagakubo, "Threedimensional bipedal stepping motion using neural oscillators - towards humanoid motion in the real world," in IEEE/RSJ International Conference on Intelligent Robots and Systems, 1998, pp. 8489.

[15] A. J. Ijspeert, "A connectionist central pattern generator for the aquatic and terrestrial gaits of a simulated salamander," Biological Cybernetics, Vol.84, pp. 331-348, 2001.

[16] K. Tsujita, K. Tsuchiya, and A. Onat, "Adaptive gait pattern control of a quadruped locomotion robot," in IEEE/RSJ International Conference on Intelligent Robots and Systems, 2001, pp. 2318-2325.

[17] M. A. Lewis et al., "An in silico central pattern generator: silicon oscillator, coupling, entrainment, and physical computation," Biological Cybernetics, Vol.88, pp. 137-151, 2003.

[18] Y. Koike, and M. Kawato, "Estimation of arm posture in 3-D space from surface EMG signals using a neural network model," Trans. Inst. Electron., Inform., Commun. Eng., Vol.J77-D-II, No.1, pp. 193-203, 1994

[19] O. Fukuda, T. Tsuji, M. Kaneko, and A. Otsuka, "A HumanAssisting Manipulator Teleoperated by EMG Signals and Arm Motions," IEEE Transactions on Robotics and Automation, Vol.19, No.2, pp. 210-222, 2003.

[20] G. Taga, "A model of the neuro-musculo-skeletal system for human locomotion II. Real-time adaptability under various constraints," Biological Cybernetics, Vol.73, pp. 113-121, 1995.

[21] N. Ogihara, and N. Yamazaki, "Generation of Human Bipedal Locomotion by a Bio-Mimetic Neuro-Musculo-Skeletal Model," Biological Cybernetics, Vol.84, pp. 1-11, 2001

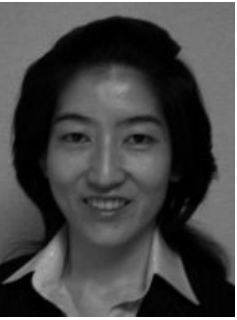

Name:

Mihoko Otake

Affiliation:

Assistant Professor, Department of Frontier Sciences and Science Integration, The University of Tokyo

Address:

5-1-5 Kashiwa-no-ha, Kashiwa-shi 277-8568, Japan

Brief Biographical History:

2003-2004 Department of Mechano-Informatics, the University of Tokyo 2004- PRESTO Researcher, the Japan Science and Technology Agency 2005- Department of Frontier Sciences and Science Integration, the University of Tokyo

\section{Main Works:}

- M. Otake, and T. Takagi, "Reassembly and Interfacing Neural Models Registered on Biological Model Databases," Genome Informatics, Vol.16, No.2, 2005 (in Press).

- M. Otake, and Y. Nakamura, "Anatomical Model of the Spinal Nervous System and its Application to the Coordination Analysis for Motor Learning Support System," In Proceedings of the IEEE International Conference on Robotic Systems, pp. 847-852, 2005.

- M. Otake, Y. Kagami, M. Inaba, and H. Inoue, "Motion design of a starfish-shaped gel robot made of electro-active polymer gel," Robotics and Autonomous Systems, Vol.40, pp. 185-191, 2002.

Membership in Learned Societies:

- The Institute of Electrical and Electronics Engineers (IEEE)

- The Robotics Society of Japan (RSJ)

- The Japanese Society for Bioinformatics (JSBI)

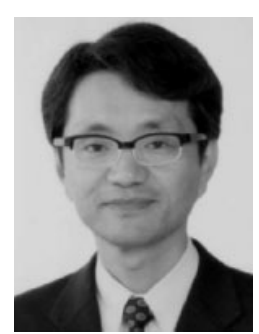

Name:

Yoshihiko Nakamura

\section{Affiliation:}

Professor, Department of Mechano-Informatics, the University of Tokyo

Address:

7-3-1 Hongo, Bunkyo-ku, Tokyo 113-8656, Japan

\section{Brief Biographical History:}

1982-1987 The Automation Research Laboratory, Kyoto University 1987-1991 Department of Mechanical and Environmental Engineering, University of California, Santa Barbara

1991- Department of Mechano-Informatics, the University of Tokyo

Main Works:

- T. Inamura, I. Toshima, H. Tanie, and Y. Nakamura, "Embodied Symbol Emergence based on Mimesis Theory," International Journal of Robotics Research, Vol.23, No.4, 2004.

- Y. Nakamura, W. Chung, and O. J. Sordalen, "Design and Control of the Nonholonomic Manipulator," IEEE Trans. on Robotics and Automation, Vol.17, No.1, pp. 48-59, February, 2001.

- Y. Nakamura, and K. Yamane, "Dynamics Computation of Structure-Varying Kinematic Chains and Its Application to Human Figures," IEEE Trans. on Robotics and Automation, Vol.16, No.2, pp. 124-134, 2000.

Membership in Learned Societies:

- The Institute of Electrical and Electronics Engineers (IEEE)

- The American Society of Mechanical Engineers (ASME)

- The Robotics Society of Japan (RSJ) 